\title{
Quantification of impact damages in CFRP and GFRP structures with thermography and ultrasonics
}

\author{
by C. Maierhofer*, R. Krankenhagen, M. Röllig*, T. Heckel**, D. Brackrock*, M. Gaal*
}

*Bundesanstalt für Materialforschung und -prüfung (BAM), Division 8.7, Richard-Willstätter-Str. 11, 12489 Berlin, Germany, christiane.maierhofer@bam.de

${ }^{*}$ Bundesanstalt für Materialforschung und -prüfung (BAM), Division 8.4, Unter den Eichen 87, 12205 Berlin, Germany, thomas.heckel@bam.de

\begin{abstract}
The extent of damage caused by impacts in fibre reinforced composites depends on the energy of the impacts, on the velocity and the shape of the impacting body, on the material and structure of the composite and on the geometry of the structure. Here, mainly the thickness of the component is essential. The non-destructive evaluation of these damages can be carried out using both ultrasound and active thermography methods. A comparison of the detection sensitivity of these methods for the different damages is carried out in this paper depending on the fibre composite material used (CFRP and GFRP), the thickness of the material and the impact energy. The NDT methods used after the damage are supplemented by thermographic measurements with high temporal resolution, which were already recorded during the impact.
\end{abstract}

\section{Introduction}

Impacts in fibre-reinforced composites can lead to different types of damage. Cracks and delaminations are generated within the matrix, fibre-matrix detachments are initiated and fibre cracks might occur. The extent of the damage depends on velocity, energy and shape of the impacting body, the composition and structure of the fibre-reinforced composites, and on the geometry of the affected component. In this case, the thickness of the structure is of particular importance. Depending on the severity and extent of the damage, it might be visible at the impacted surface and could have an influence on the long-term reliability of the component [1].

For safety-relevant components, all known impacts, whether with or without visible damage, must therefore be inspected using non-destructive testing methods and characterised at least in terms of lateral expansion. The last aspect is particularly important for impacts with visible damages as well, as the visual inspection of a surface does not allow any conclusions to be drawn about the lateral extent of the damage inside the structure. Both, active thermography and ultrasonics are very well suited for locating and characterizing impact damages after impact load [2, 3, 4]. Depending on the respective excitation techniques, different penetration depths and spatial resolutions can be achieved with both methods. Results such as the lateral extent of damage can be depicted in depth resolved form. In addition, thermographic methods allow also the observation of the thermal signature of an impact during and after load (passive thermography) [5].

As part of the EMRP project VITCEA [6], various fibre-reinforced composites with glass fibres, carbon fibres, different matrix systems and different thicknesses were systematically damaged with impacts (low velocity) with varying energies and investigated during and after the damage using the above-mentioned methods. The paper describes the advantages and disadvantages of the respective excitation techniques of active thermography and ultrasonics and compares them with the in-situ measurement results of passive thermography. It will be shown that the damages proven by non-destructive testing after the impact deviates from the thermal active areas of the component being detected during the impact.

\section{Experimentals}

\subsection{Test specimen and impact loading}

Within the project VITCEA, various CFRP and GFRP materials were cured in autoclaves with documented temperature and pressure progressions [7]. These materials consist of quasiisotropic fibre orientations and have thicknesses from 2.4 to $5.0 \mathrm{~mm}$. For the CFRP laminates described below, unidirectional prepregs with carbon fibres and epoxy matrix were laid up in a quasiisotropic orientation. The test coupons with $8([+45 / 0 /-45 / 90] 2 s)$ and 16 ([+45/0/$45 / 90] 4 \mathrm{~s}$ ) layers of prepreg correspond to material thicknesses of 2.4 and $4.8 \mathrm{~mm}$, respectively. The curing occurred at temperatures of up to $120^{\circ} \mathrm{C}$ and pressures of up to 6 bar for a period of about $160 \mathrm{~min}$. GFRP specimens were produced with different fibre and matrix systems. In the following, only the results of a GFRP material consisting of a quadaxial fabric and an epoxy matrix are presented. Here, 5 and 8 layers of the quasiisotropic fabric $\left(0 \%-45^{\circ} / 90^{\circ} / 45^{\circ}\right)$ were laid on top of each other, whereby the uppermost layer has a $0^{\circ}$ orientation and the lowermost layer has $\mathrm{a}+45^{\circ}$ orientation. However, the $0^{\circ} / 90^{\circ}$ orientations have a linear density (weight per unit length) which is twice as high as the $+45^{\circ} \%-45^{\circ}$ orientations. These samples were cured at $60^{\circ} \mathrm{C}$. Plates with sizes of $240 \times 100 \mathrm{~mm} 2$ have been used for the impact tests. 
Table 1. Examined CFRP and GFRP specimens with plate thickness, impact energy, material system, layer arrangement and experimentally determined thermal diffusivity perpendicular to the surface.

\begin{tabular}{|c|c|c|c|c|c|}
\hline $\begin{array}{l}\text { test } \\
\text { specimen }\end{array}$ & $\begin{array}{l}\text { thickness } \\
\text { in } \mathrm{mm}\end{array}$ & fibre and matrix system & layer orientation & $\begin{array}{l}\text { diffusivity in } \\
\mathrm{mm}^{2} / \mathrm{s}\end{array}$ & energy in $\mathbf{J}$ \\
\hline $\begin{array}{l}\text { AFMF } \\
\text { (CFRP) }\end{array}$ & 2.4 & \multirow{2}{*}{$\begin{array}{l}\text { Gurit SE84LV carbon fibre/ } \\
\text { epoxy UD prepregs with } \\
\text { T700 fibre, thickness of } \\
\text { one layer: } 0.3 \mathrm{~mm}\end{array}$} & $\begin{array}{l}8 \text { layers }[+45 / 0 / \\
-45 / 90]_{\mathrm{s}}\end{array}$ & \multirow{2}{*}{$\begin{array}{r}0.0039 \pm \\
0.0002\end{array}$} & $1.0 ; 2.0 ; 4.0 ; 8.0$ \\
\hline $\begin{array}{l}\text { 1AFMG } \\
\text { (CFRP) }\end{array}$ & 4.8 & & $\begin{array}{l}16 \text { layers [+45/0/ } \\
-45 / 90]_{2 \mathrm{~s}}\end{array}$ & & $\begin{array}{l}2.0 ; 4.0 ; 5.0 ; 6.0 ; \\
6.8 ; 7.5 ; 15.0\end{array}$ \\
\hline $\begin{array}{l}\text { AFPD } \\
(\text { GFRP) }\end{array}$ & 3.2 & \multirow{2}{*}{$\begin{array}{l}\text { GFRP glass } 20 \mathrm{LV} / \\
\text { quadaxial Formax fabric } \\
\text { FGE111, } \\
\text { Prime } 20 \mathrm{LV} \text { epoxy } \\
0^{\circ} / 90^{\circ}: 600 \text { tex } \\
-45^{\circ} / 45^{\circ}: 300 \text { tex }\end{array}$} & $\begin{array}{l}5 \text { layers of fabric, within } \\
\text { one layer } \\
0 \%-45^{\circ} / 90^{\circ} / 45^{\circ}\end{array}$ & \multirow{2}{*}{$\begin{array}{r}0.0026 \pm \\
0.0002\end{array}$} & $\begin{array}{l}1.0 ; 2.0 ; 3.0 ; 5.0 ; \\
8.0 ; 15.0\end{array}$ \\
\hline $\begin{array}{l}\text { 1AFPC } \\
\text { (GFRP) }\end{array}$ & 5.0 & & $\begin{array}{l}8 \text { layers of fabric, within } \\
\text { one layer } \\
0^{\circ} /-45^{\circ} / 90^{\circ} \%+45^{\circ}\end{array}$ & & $\begin{array}{l}3.5 ; 7.5 ; 13.5 ; \\
15.0\end{array}$ \\
\hline
\end{tabular}

Impact loads were introduced with a drop impact tester according to DIN ISO 18352:2017-11, but with deviations concerning the diameter of the impact body $(12 \mathrm{~mm}$ instead of $8 \mathrm{~mm}$ ) and the mounting of the test specimen (an open frame instead a closed frame and a two-point fastening instead of a four-point fastening), see Fig. 1.a. The drop tower was not instrumented, i. e. the velocity and force of the drop weight were not determined experimentally, but were calculated from the mass and the drop height. The impact energy was varied from 0.9 to $16 \mathrm{~J}$ using two different impact masses of $627 \mathrm{~g}$ and $1287 \mathrm{~g}$ and different drop heights from $16 \mathrm{~cm}$ to $130 \mathrm{~cm}$. The velocities of the falling weights were between 1.7 $\mathrm{m} / \mathrm{s}$ and $5.0 \mathrm{~m} / \mathrm{s}$, thus low velocity impacts were realized. Table 1 summarises the investigated test specimens with the name of the test specimens, the impact energies, the material, the layer arrangement and the thermal diffusivity determined from active thermography measurements with flash excitation in transmission configuration. The emissivities of the surfaces were measured as described in [7]. Here, within the spectral sensitivity of the infrared (IR) camera described below values of 0.86 and 0.95 were obtained for CFRP and GFRP, respectively.

a)

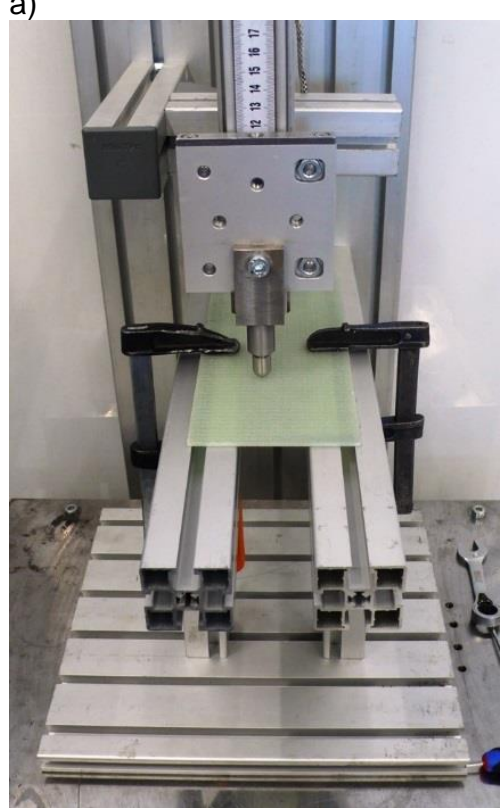

b)

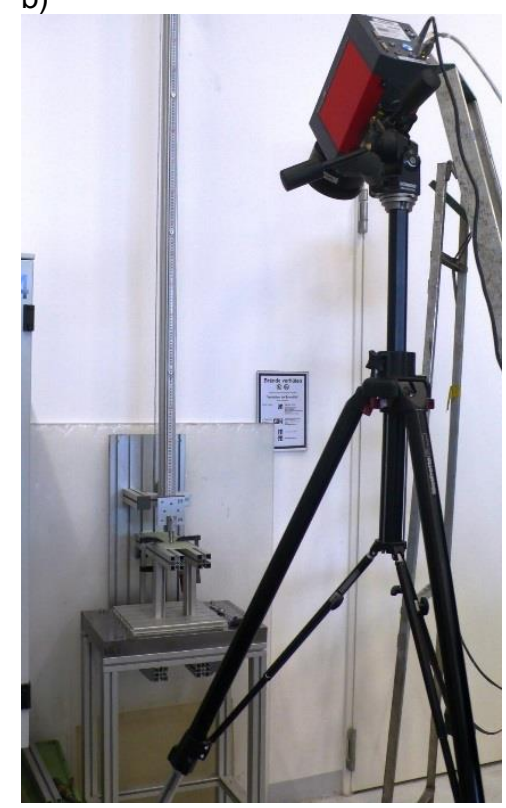

c)

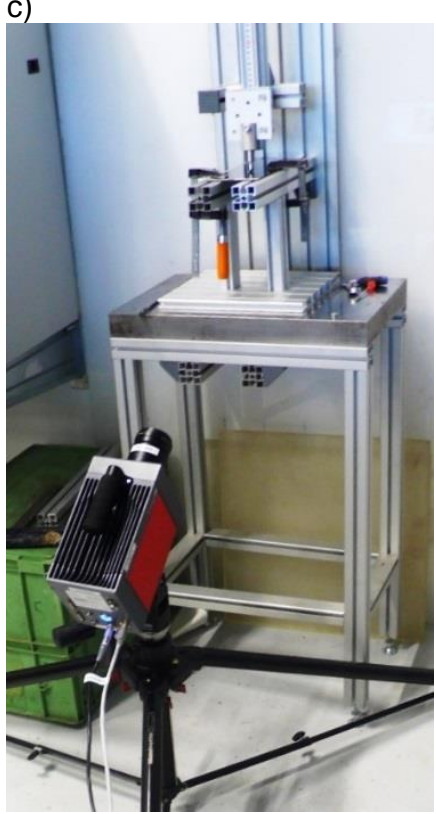

Fig. 1. Experimental set-up for (a) impact loads, (b) passive thermography of the impact side of the test specimen during impact load, (c) passive thermography from the rear side of the test specimen during impact load.

\subsection{Passive thermography}

The main idea of the in-situ observation was to detect the damaged areas by means of the energy release during the plastic deformation. The spatial and temporal temperature distribution of the test specimens was recorded either on the side of the impact (front side) or on the opposite side of the impact (rear side), see Fig. 1.b and 1.c, respectively. The IR camera has a cooled InSb detector with a size of $640 \times 512$ pixels, a sensitive wavelength range of 2 to $5 \mu \mathrm{m}$ and a thermal resolution (NETD) of about $30 \mathrm{mK}$. In order to achieve a high frame rate of $800 \mathrm{~Hz}$ and thus a high temporal resolution of $1.25 \mathrm{~ms}$, the IR camera was operated in windowing mode with an image size of $160 \times 128$. A temperature measuring range of $-10^{\circ} \mathrm{C}$ to $60^{\circ} \mathrm{C}$ was selected, corresponding to an integration time of $1.12 \mathrm{~ms}$. For the recording of 
thermograms during impact load, the IR camera has been positioned either at the front or at the rear side of the test specimens. Measurements at the front side were performed only for the CFRP and the thin GFRP test specimens. The measurements from both sides of the specimen were carried out with lenses of different field of views and the IR camera had to be tilted at an angle of $50^{\circ}$ to the surface normal. As a result, the spatial resolution of the thermograms is different in $x$ - and $y$-direction and different at both sides. Table 2 summarizes the spatial resolutions of the measurement methods used.

Table 2. Spatial resolution of the different methods.

\begin{tabular}{|l|r|r|}
\hline method & $\begin{array}{l}\text { spatial resolution in x- } \\
\text { direction in mm/pixel }\end{array}$ & $\begin{array}{l}\text { spatial resolution in y- } \\
\text { direction in mm/pixel }\end{array}$ \\
\hline $\begin{array}{l}\text { passive thermography, } \\
\text { measurements at the front side of } \\
\text { the CFRP test specimens }\end{array}$ & 0.21 & 0.32 \\
\hline $\begin{array}{l}\text { passive thermography, } \\
\text { measurements at the rear side of } \\
\text { all test specimens }\end{array}$ & 0.35 & 0.57 \\
\hline $\begin{array}{l}\text { active thermography with flash } \\
\text { and pulse excitation }\end{array}$ & 0.23 & 0.23 \\
\hline air-coupled ultrasonics & 0.5 & 0.5 \\
\hline ultrasonics with matrix array & 1.0 & 1.0 \\
\hline
\end{tabular}

\subsection{Active thermography}

For active thermography, two different excitation techniques were applied:

- $\quad$ Flash excitation with four synchronized flash lamps with a $2.6 \mathrm{~ms}$ long flash and a total converted energy of $24 \mathrm{~kJ}$, see Fig. 2.a. To reduce the thermal afterglow of the lamps, polymethyl methacrylate (PMMA) plates were mounted between the flash lamps and the test specimens. Here, an energy density of about $6,550 \mathrm{~J} / \mathrm{m}^{2}$ was brought to the surface of the test specimens.

- $\quad$ Pulse excitation with an IR radiator with a power of $2.4 \mathrm{~kW}$ (not shown here). The test specimens were heated for $15 \mathrm{~s}$. During this time, the IR radiator was moved evenly over the surface of the specimen at a distance of about $10 \mathrm{~cm}$ to the surface. A total energy density of about $32,760 \mathrm{~J} / \mathrm{m}^{2}$ was brought to the surface.

These measurements were carried out in reflection configurations at both sides of the test specimens and in transmission configuration. The surface temperature was recorded with an IR camera with an InSb detector with a size of $1280 \times 1024$ pixels, a sensitive wavelength range of 2 to $5 \mu \mathrm{m}$, an NETD of $30 \mathrm{mK}$, frame rates of $50 \mathrm{~Hz}$ for flash excitation and $10 \mathrm{~Hz}$ for excitation with the IR radiator, and for each data set with a sequence length of 3000 images. For GFRP, it must be considered that the material is partially translucent in the visible and near-infrared spectral range, i. e. within the spectrum of excitation sources. For this reason, not all the radiation is absorbed at the surface, thus a part of the radiation penetrates the specimen and is partially absorbed directly at the surface of the defect. For the measurements presented in the following, the sample surfaces were not coated.

a)

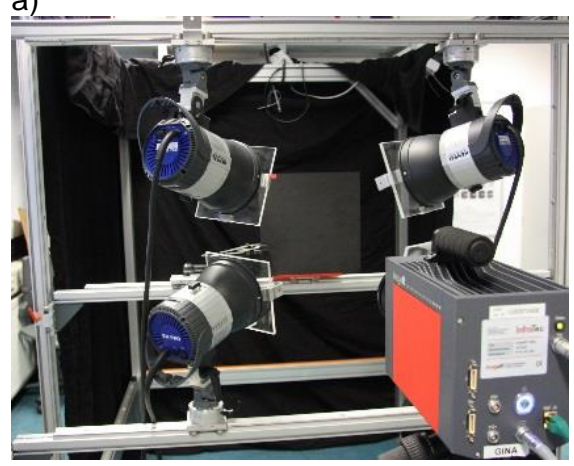

b)

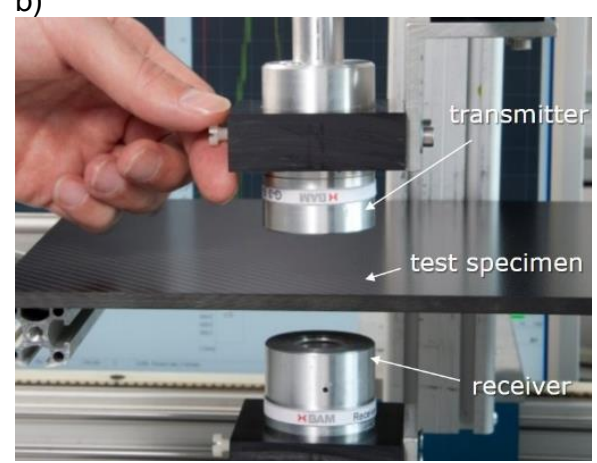

c)

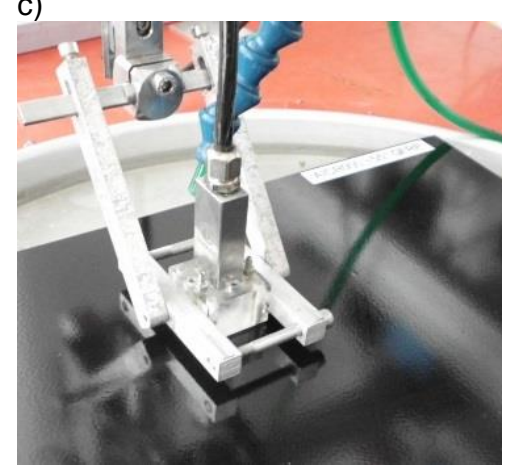

Fig. 2. Experimental set-up for (a) active thermography with flash excitation in reflection configuration, (b) air-coupled ultrasonics in transmission configuration, (c) ultrasonic phased array with contact to the test specimen. 


\subsection{Air-coupled ultrasonics}

The measurements with air-coupled ultrasonics were carried out only in transmission configuration, i. e. the transducers were located at opposite sides of the test specimens, see Fig. 2.b. Two probes developed at BAM [4] with a mid frequency of $260 \mathrm{kHz}$, an aperture of $27 \mathrm{~mm}$ and a radius of curvature of $50 \mathrm{~mm}$ were used. The transducers were aligned so that the received signal was maximum and the focus of the transducers was in the plane of the specimen. Both transducers were moved parallel and automatically by a scanning unit and were stopped for the measurements. The distance between each measurement point was $0.5 \mathrm{~mm}$, and the distance between each track was $0.5 \mathrm{~mm}$. Defects like delaminations are leading to reflections of ultrasonic waves and thus to a damping of the transmitted ultrasonic intensity. Here, the scaling of the transmitted intensity is done inversely to that of the reflected intensity detected with the ultrasonic phased array.

\subsection{Ultrasonic phased array}

Ultrasonic phased array measurements were realized with a matrix array of Sonaxis with $10 \times 6$ elements and a mid frequency of $2.25 \mathrm{MHz}$ in reflection configuration. The phased array was focused to a depth of $2.4 \mathrm{~mm}$. The probe was scanned automatically with a mechanised manipulator system and using water coupling, see Fig. 2.c. The distance between the measurement points was $1 \mathrm{~mm}$ in each direction. Defects like delaminations can either be detected directly by the reflected wave, or indirectly by a shadowing of the backwall reflection.

\section{Results}

\subsection{Passive thermography}

For evaluating the front and rear side thermograms recorded during the impact, a zero thermogram at the beginning of the sequence, i. e. a thermogram before the impact, was first subtracted from the entire sequence. This reduces disturbing influences caused by the direct reflection of the IR camera, variations in surface emissivity or possible small temperature gradients. Subsequently, the temperature in each thermogram was averaged around the position of the impact in the range of $6 \times 6$ pixels. This value was recorded over time for the whole sequence. Fig. 3 shows these temperature-time diagrams for the front and rear side of the thin CFRP test specimen AFMF for different impact energies.

The temperature development at the front side in Fig. 3.a shows an immediate temperature increase during and after the impact. The temperature maximum is increasing nearly linearly by doubling the impact energy. In addition, it is observed that for higher impact energies, the maximum temperature was reached later and the cooling down occurred slower.

At the rear side (see Fig. 3.b), it was observed that during impact, within the measurement area around the impact point the test specimens are initially cooling down. However, since the temporal resolution of the IR camera is limited, only one to two data points were recorded during this cooling down. Thus, a quantitative comparison of the cooling down depending on the impact energy is not possible. This cooling can be explained by the thermoelastic effect. The rear side of the specimen is first elastically tensioned during impact before plastic deformations occur. The plastic deformation can be recognized by the significant rise in temperature after impact. Again, the maximum temperature increases approximately linearly while doubling the impact energy up to an energy of $4 \mathrm{~J}$, but is much lower than at the front side. For an impact energy of $8 \mathrm{~J}$, the temperature at the rear side increases significantly stronger than for the lower energies, which can be related to much larger damages. For $8 \mathrm{~J}$, a temperature maximum similar as at the front side was reached. For all impacts, the temperature rises at the rear side decreased much slower than at the front side.

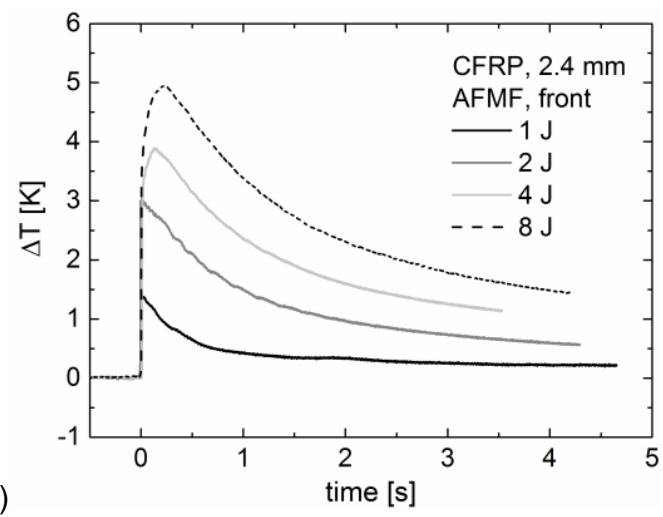

a)

Fig. 3. Temperature increase during impact load with different energies at the surface of the CFRP AFMF test specimens with a thickness of $2.4 \mathrm{~mm}$ recorded at the front side (a) and at the rear side (b).

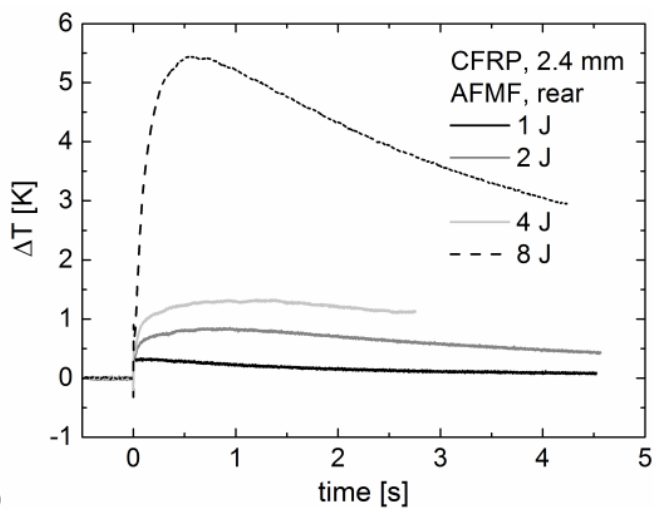

b) 
In Fig. 4, the temperature rises at the rear sides of the thin CFRP samples are compared with those of the thick CFRP samples, which are twice as thick as the thin samples. For the thin samples, results for impact energies of $1 \mathrm{~J}, 2 \mathrm{~J}$, and $4 \mathrm{~J}$, and for the thick samples of $5 \mathrm{~J}, 8 \mathrm{~J}$, and $15 \mathrm{~J}$ are shown. For the thick CFRP samples, impact energies below $5 \mathrm{~J}$ did not result in a measurable temperature increase at the rear side, therefore these data are not shown. Fig. 4 shows that similar maximum temperatures for the thick samples can be observed at impact energies which are about four to five times larger than for the thin samples. In addition, it is noticeable that the temperature rises of the thin specimens decreases more quickly with time than of the thick specimens.

High-contrast thermograms from the rear sides of the test specimens appear approx. $56 \mathrm{~ms}$ after each impact. These are shown in Fig. 5 for the thin and thick CFRP test specimens at different impact energies. For a certain sample thickness, it is easy to see how the extent of the damage increases with increasing impact energy. The damage is elongated parallel to the $45^{\circ}$ orientation, which corresponds to the orientation of the lowermost prepreg layer. The warm middle line can be correlated to a matrix crack, which is also visible on the surface. The slightly less warm areas next to this matrix crack can probably be assigned to delaminations between the prepreg layers. These seem to be confined by $0^{\circ}$ and $90^{\circ}$ orientations. For impact energies with comparable temperature rise of the thin and thick test samples, the damaged areas of the thicker samples are larger. Here, several parallel matrix cracks can be observed especially at higher impact energies.
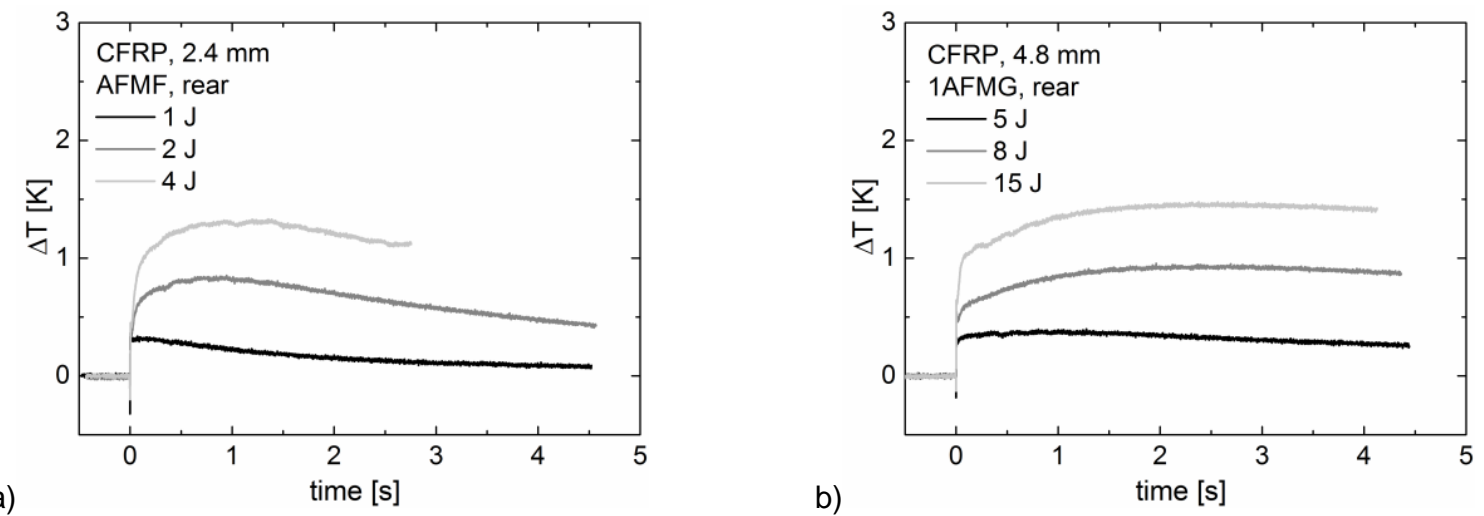

Fig. 4. Temperature increase during impact load for different energies at the rear side of the CFRP AFMF test specimens with a thickness of $2.4 \mathrm{~mm}$ (a) and at the rear side of the 1AFMG test specimens with a thickness of $4.8 \mathrm{~mm}(b)$.

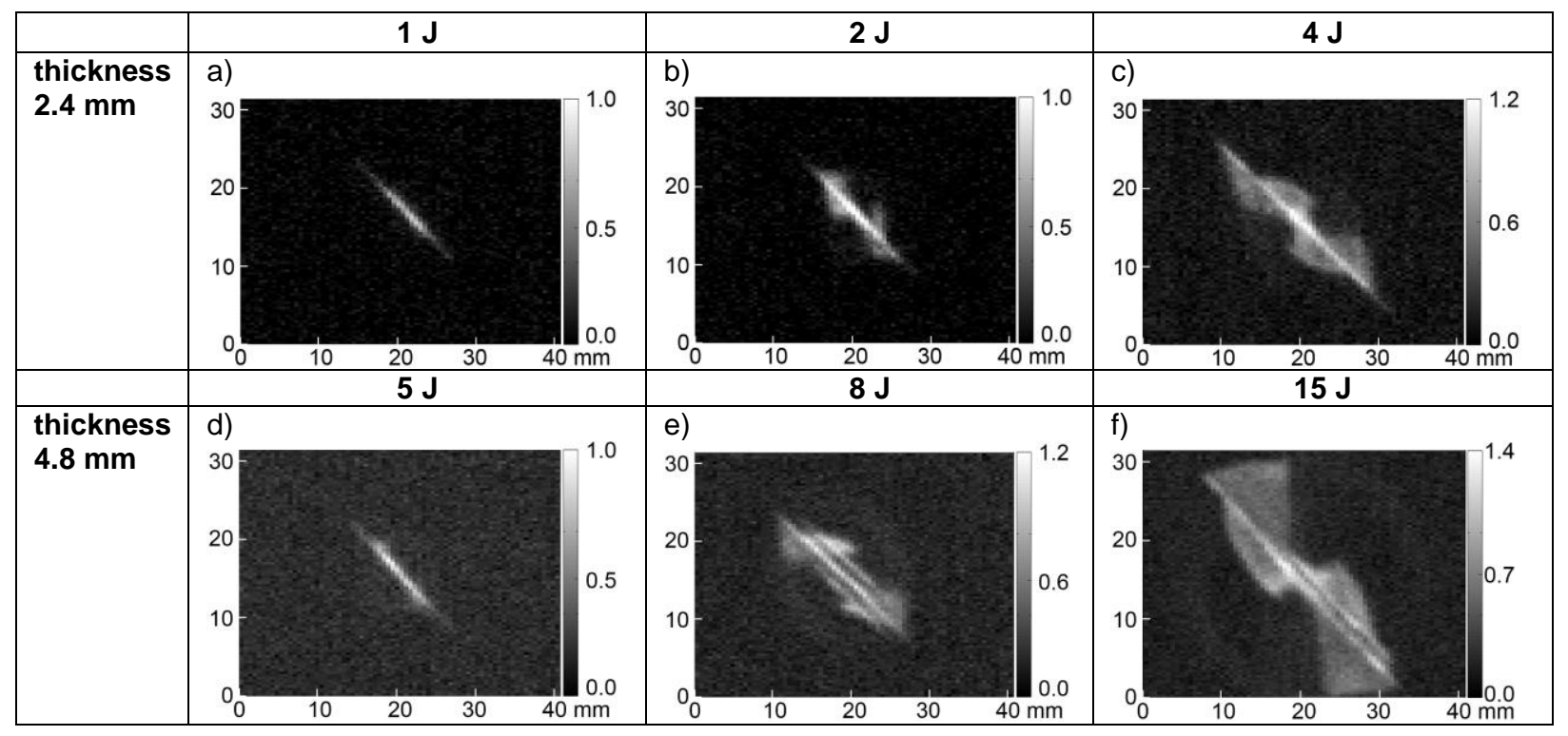

Fig. 5. Thermograms (each recorded $56 \mathrm{~ms}$ after the impact) at the rear side of the thin (top) and thick (bottom) CFRP test specimens for different impact energies. Units of the grey scale: $K$ 


\subsection{Comparison of methods}

A realistic application case of NDT methods for the determination of damage size and depth is in reflection configuration from the side of the impact. In most cases, structures and components to be tested will only be accessible in this alignment. But this study is focussing on those measurement configurations of the applied methods, which are giving information about the maximum extend of the damage. Thus, the thermographic testing of CFRP by flash excitation was applied at the rear side of the samples because the penetration depth of the flash method is limited and the main damage occurs in the lower half of the panel thickness. Furthermore, air-coupled ultrasonics can actually only be performed in transmission configuration, i.e. access from both sides of the specimen was required. It is clear that a comparison of such different methods is not a real evaluation of all methods under similar conditions, but gives some basic knowledge about the advantages and disadvantages of each technique.

The configurations to be compared are:

- $\quad$ Flash thermography after impact: reflection configuration from the rear side

- Ultrasonics with matrix array: reflection configuration from the front side showing either the direct reflection from the impact damage or the damage induced shadowing of the backwall echo

- $\quad$ Air-coupled ultrasonics: transmission configuration.

Additionally, the results of passive thermography are included as reference.

In Figs. 6 and 7, the results of the different test methods are compared for the $4 \mathrm{~J}$ impact of the thin CFRP test specimen and for the $13.5 \mathrm{~J}$ impact of the thick GFRP test specimen. The origin of the images is described in each caption.

First of all, a comparison of the results of only the thermographic methods for the thin CFRP test specimen shows that most of the information about the extent of the damage can be taken from measurements made during the impact with passive thermography, see Fig 6.a. Here, matrix cracks and probable delaminations between the laminate layers can be clearly distinguished from each other, and the extent of the damage appears larger as within the thermograms recorded with flash and impulse excitation after the damage. A comparison of the results of active thermography for the two different materials shows that in case of the thin CFRP test specimen an impact damage with clearly defined edges was observed (crack and delamination) after the flash excitation. In case of the GFRP test specimen more details within the damaged area could be detected (cooler area inside) after $15 \mathrm{~s}$ impulse excitation, see Fig. 7.c. For the GFRP test specimen, the thermograms recorded with active thermography after the damage show a larger extent of the damage than the thermograms taken with passive thermography during the impact load. This is probably due to the different causes of the respective thermal contrasts: active thermography is sensitive to variations of the heat flow inside the specimen after surface heating, while passive thermography during the impact detects thermal contrasts resulting from a superposition of heat release and subsequent heat flow from different depths of the entire specimen volume.

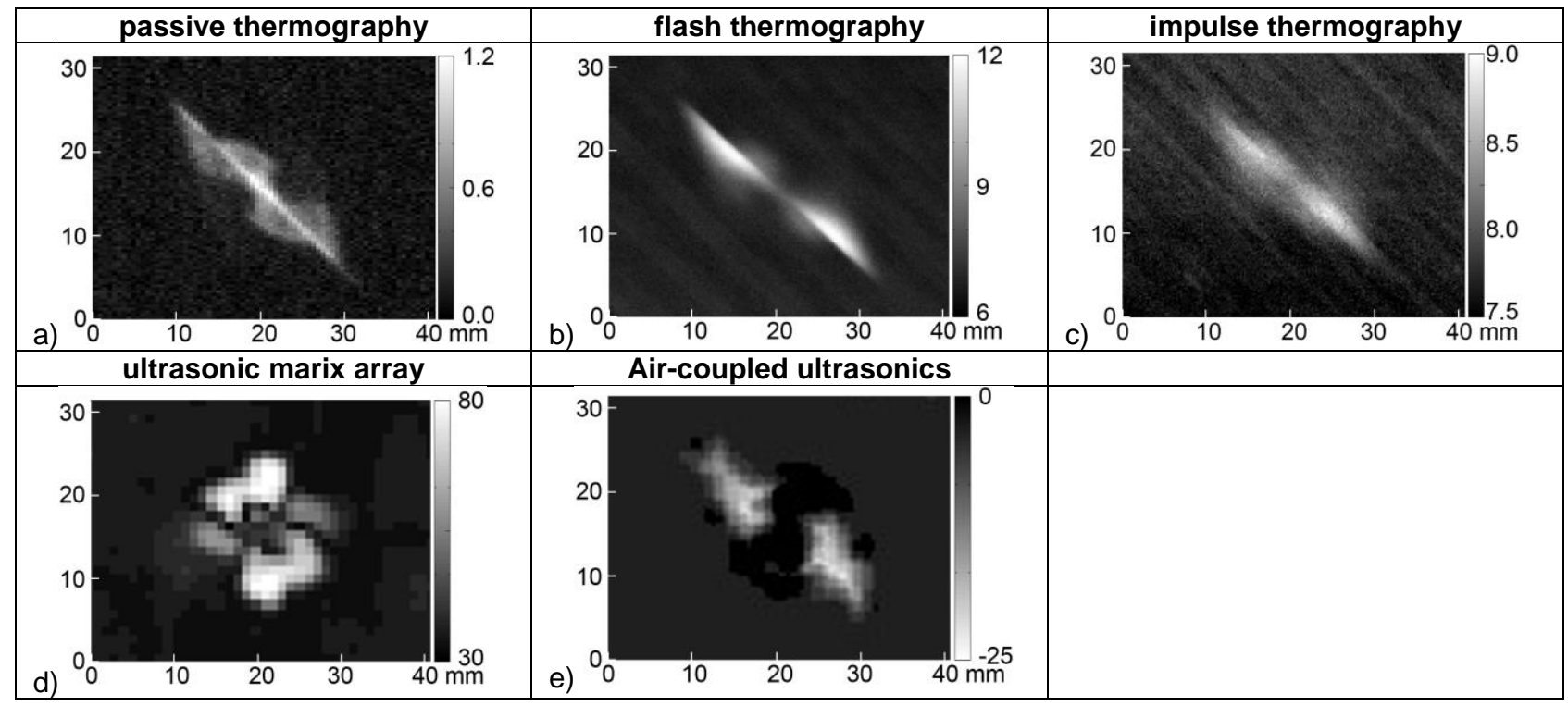

Fig. 6. Comparison of results of different methods obtained at the AFMF CFRP test specimen with a thickness of $2.4 \mathrm{~mm}$ loaded with an impact energy of $4 \mathrm{~J}$. a) Thermogram recorded $56 \mathrm{~ms}$ after the impact (passive thermography).

b) Thermogram recorded $100 \mathrm{~ms}$ after the flash (flash thermography). c) Thermogram recorded directly after $15 \mathrm{~s}$ heating with an IR radiator. d) $C$-scan obtained with the ultrasonic matrix array focused to the middle of the specimen and taken from a depth close to the rear side. e) $C$-scan of the air-coupled ultrasonics. Units of the grey scales of the thermograms: $K$. Units of the grey scales of the $C$-scans: $d B$.

The C-scan recorded with the ultrasonic matrix array on the thin CFRP specimen (see Fig. 6.d) was taken from a depth slightly before the backwall, thus it contains the direct reflection from the impact damage. It shows a completely 
different structure of the impact damage than the thermographic methods and also than the air-coupled ultrasonic method (see Fig. 6.e). While the middle of the defect hardly produces any reflections, elongated areas with higher reflection intensities appear parallel to the $+45^{\circ}, 90^{\circ}$ and $0^{\circ}$ fibre orientations. The transmission patterns of the air-coupled ultrasonics (Fig. 6.e) show a strong attenuation of the signal parallel to the $+45^{\circ}$ orientation and thus resembles the results shown in the thermograms. The circular dark structure around the impact centre means that the transmission of the ultrasound is even better than in the undisturbed area. This effect could be attributed to the fact that in this area the material was compacted by the impact. With both ultrasonic methods, the crack could not be detected directly.

The results of the two ultrasonic methods on the thick GFRP test specimen show a comparable outer geometry of the impact damage. The C-scan with the matrix array recorded the reflections from the backwall, therefore the obtained signal attenuation it is a superposition of all delaminations in all depths, which cause a corresponding attenuation by absorption and scattering of the ultrasonic waves. More details can be seen in the transmission pattern of the air-coupled ultrasonic method inside the defect. Here, a ring-shaped structure with higher transmission is visible. The reason is unclear, but it is worth to note the circular shaped structure observed by impulse thermography in the same region. The ultrasonic results show a damage extent similar to that recorded with active thermography.

\begin{tabular}{|c|c|c|}
\hline passive thermography & flash thermography & impulse thermography \\
\hline 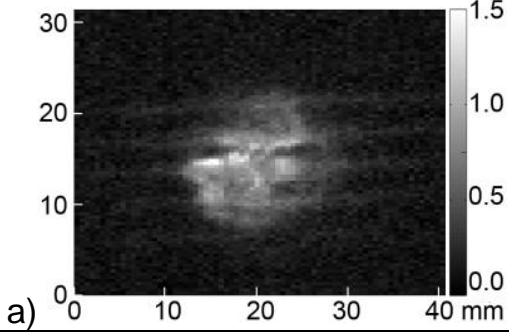 & 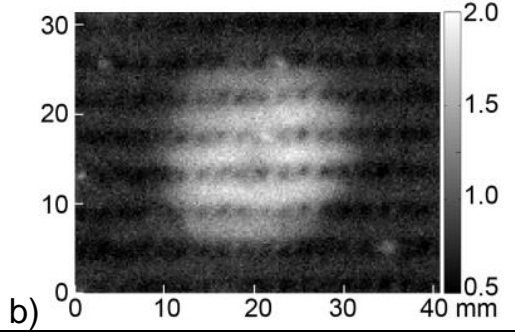 & 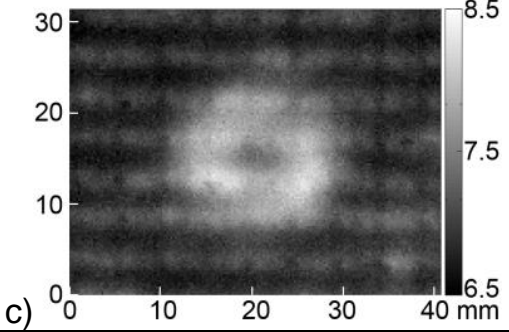 \\
\hline ultrasonic marix array & air-coupled ultrasonics & \\
\hline 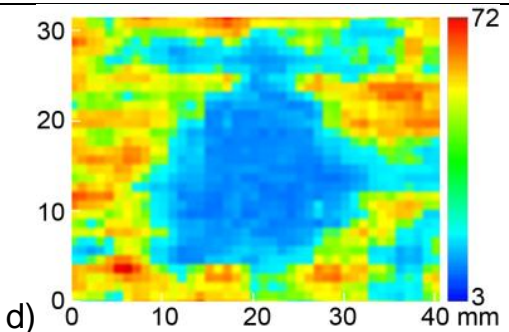 & e) & \\
\hline
\end{tabular}

Fig. 7. Comparison of results of different methods obtained at the 1AFPC GFRP test specimen with a thickness of $5.0 \mathrm{~mm}$ loaded with an impact energy of $13.5 \mathrm{~J}$. a) Thermogram recorded $56 \mathrm{~ms}$ after the impact (passive thermography). b) Thermogram recorded $100 \mathrm{~ms}$ after the flash (flash thermography). c) Thermogram recorded directly after $15 \mathrm{~s}$ heating with an IR radiator. d) $C$-scan obtained with the ultrasonic matrix array focused to the middle of the specimen and taken from the depth position of the backwall. e) C-scan of the air-coupled ultrasonics. Units of the grey scales of the thermograms: $K$. Units of the grey/colour scales of the $C$-scans: $d B$.

\section{Conclusion and outlook}

The investigations of the impact damaged CFRP and GFRP test specimens with thermography and ultrasonics show that kind and shape of the damage for the CFRP and GFRP are quite different: for the CFRP test specimens, a defined large crack can be detected which has spread parallel to the direction of the fibres of the lowermost layer of the laminate. For impact energies higher than $1 \mathrm{~J}$ for the thinner samples and higher than $5 \mathrm{~J}$ for the thicker samples, delaminations occur which have their largest extents between the impact point and each crack tip. With increasing impact energy, the length of the cracks and the area of the delaminations are increasing as well. For the GFRP test specimen, a circular shaped area centred around the impact point can be detected, which might be related to delaminations. In addition, the $0^{\circ}$-orientation of the lowermost glass fibre layer can be detected and in between and parallel to these fibres and within the circular area, some small hot lines can be seen. These might be originated to small matrix cracks and/or fibre-matrix separations.

The thermograms recorded during the impacts at the rear side of the test specimens show the most detailed information on this damage evolution. These data show first a cooling down due to thermoelastic bending, followed by a sudden temperature increase generated by the cracks and a slower and less temperature increase generated by the delaminations. As the heat is directly generated at the depth and position of damage, more detailed contrasts are obtained than with active thermography.

Using active thermography with flash and with impulse excitation, the thermograms show a less detailed structure of the damages than those thermograms recorded with passive thermography during the impact. For the CFRP test specimens, the introduced optical radiation is first absorbed at the surface of the specimen followed by heat diffusion. 
Above the areas with delaminations, the heat is accumulating resulting in a temperature increase at the surface. The extent of the damage appears smaller than with passive thermography. The GFRP test specimens are partially translucent within the wavelength range of the optical excitation, therefore most of the radiation is directly absorbed at the delaminations. Here, the extent of the damage appears larger as in the thermograms recorded passively during the impact.

Ultrasonic waves are directly reflected and scattered at the delaminations induced by the impact. Thus, the delaminations could be detected either by direct reflections or by shadowing of the backwall reflection in reflection configuration, or by the damping of a transmitted signal in transmission configuration. Concerning the extent of the damage, the results of the measurements with the ultrasonic methods are comparable to the results of the active thermography methods recorded after the impact. An exception to this are the measurement results with the ultrasonic matrix array on the thin CFRP specimen: here, clear reflectors along three fibre orientations were observed, which could not be detected with the other methods.

Both, ultrasonic and thermographic techniques have their advantages and disadvantages. Passive thermography gives the most detailed information about the damage. However, it can only be used in planned tests to characterise defined impact loads. Active thermography can be performed without any contact to the test specimen and gives direct images, but has a limited penetration depth. Air-coupled ultrasonics can be carried out without any contact, too, but only in transmission configuration and scanning is required. And ultrasonic phased array can only be implemented with a direct coupling to the surface of the test specimens and with scanning, but can be applied in reflection configuration from the front side and has a deep penetration into the structure.

Future studies and publications will show results from further FRP materials and will focus more deeply on the comparison of thermography from the front and from the rear side.

\section{Acknowledgements}

The test specimens were prepared by M. Gower, G. Baker, M. Lodeiro and A. Aktas of the National Physical Laboratory (NPL), Teddington, UK. The impact loads were introduced together with Block Materialprüfungsgesellschaft $\mathrm{mbH}$, Berlin, Germany, with the help of F. Hohlstein. The EMRP project was jointly funded by the EMRP participating countries within EURAMET and the European Union under the project no. ENG57 VITCEA.

\section{REFERENCES}

[1] Abrate, S. Impact Engineering of Composite Structures. CISM Courses and Lectures, vol. 526. Springer, Wien, New York, 2011

[2] Maierhofer, C., Myrach, P., Reischel, M., Steinfurth, H., Röllig, M., Kunert, M., Characterising damage in CFRP structures using flash thermography in reflection and transmission configurations. Composites Part B 57 (2014), 35-45.

[3] Meola, C. et al., Nondestructive evaluation of carbon fibre reinforced composites with infrared thermography and ultrasonics, Composite Structures 134 (2015) 845-853

[4] Gaal, M. et al, Air-coupled ferroelectret ultrasonic transducers applied to testing of fiber-reinforced polymers. $2^{\text {nd }}$ International Conference of the Slovenian Society for Non-Destructive Testing, Portorož, Slovenia, 2013, http://www.ndt.net/article/ndt-slovenia2013/papers/43.pdf

[5] Meola, C., Boccardi, S., Carlomagno, G.M. et al, Impact damaging of composites through online monitoring and non-destructive evaluation with infrared thermography. NDT\&E Int. 85 (2017) 34-42.

[6] VITCEA: Validated Inspection Techniques for Composites in Energy Applications. EMRP-Project ENG57, http://projects.npl.co.uk/vitcea/

[7] Maierhofer, C., Krankenhagen, R., Röllig, M. et al, Defect characterisation of tensile loaded CFRP and GFRP laminates used in energy applications by means of infrared thermography. Quantitative InfraRed Thermography Journal Quantitative InfraRed Thermography Journal, 15:1 (2017), 17-36, DOI:

10.1080/17686733.2017.1334312 